Canadian

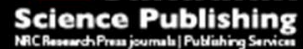

Canadian Journal of Microbiology Revue canadienne de de microbiologie

\title{
Role of a Vibrio cholerae exo-chitinase ChiA2 in Horizontal Gene Transfer
}

\begin{tabular}{|r|l|}
\hline Journal: & Canadian Journal of Microbiology \\
\hline Manuscript ID & cjm-2015-0556.R1 \\
\hline Manuscript Type: & Article \\
\hline Date Submitted by the Author: & 30 -Oct-2015 \\
\hline Complete List of Authors: & $\begin{array}{l}\text { Mondal, Moumita; National Institute of Cholera and Enteric Diseases, } \\
\text { Biochemistry } \\
\text { Chatterjee, Nabendu; National Institute of Cholera and Enteric Diseases, } \\
\text { Biochemistry }\end{array}$ \\
\hline Keyword: & V. cholerae chitinase, ChiA2, chitin, horizontal gene transfer, tfoX \\
\hline
\end{tabular}


1 Manuscript ID: cjm-2015-0556

2

3 Role of a Vibrio cholerae exo-chitinase ChiA2 in Horizontal Gene Transfer

4

5 Moumita Mondal $^{\mathrm{a}}$ and Nabendu Sekhar Chatterjee $\mathrm{a}^{\mathrm{a}^{*}}$

6

$7{ }^{\mathrm{a}}$ Division of Biochemistry, National Institute of Cholera and Enteric Diseases, Kolkata-

8700010, India.

9

10 *Corresponding author

11 Nabendu Sekhar Chatterjee

12 Division of Biochemistry, National Institute of Cholera and Enteric Diseases

13 P33 C.I.T. Road, Scheme XM, Beliaghata, Kolkata - 700 010, India

14 Phone: +91(33) 2363-3863

15 Fax: +91(33) 2363-2398

16 e-mail:nschatterjee@rediffmail.com; chatterjeens@icmr.org.in

17

18

19

20

21

22

23 


\section{Abstract}

26 Vibrio cholerae exo-chitinase ChiA2 play a key role in acquisition of nutrients by chitin 27 hydrolysis in the natural environment as well as in pathogenesis in the intestinal milieu. In 28 this study we demonstrate the importance of ChiA2 in horizontal gene transfer in the natural 29 environment. We found the expression of ChiA2 and TfoX, the central regulator of $V$. 30 cholerae horizontal gene transfer, varied with changes in environmental conditions. The 31 activity of ChiA2 was also dependent on these conditions. In three different environmental 32 conditions tested here, we observed that the supporting environmental condition for the 33 maximum expression and activity of ChiA2 was at $20^{\circ} \mathrm{C}, \mathrm{pH} 5.5$ and $100 \mathrm{mM}$ salinity in 34 presence of chitin. The same condition also induced TfoX expression and was favorable for horizontal gene transfer in $V$. cholerae. HPLC analysis showed ChiA2 released significant amount of $(\mathrm{GlcNAc})_{2}$ from chitin hydrolysis under the favorable condition. We hypothesized that under the favorable environmental condition, ChiA2 was up-regulated and maximally active to produce significant amount of $(\mathrm{GlcNAc})_{2}$ from chitin. The same environmental condition also induced tfo $X$ expression followed by its translational activation by (GlcNAc) 2

40 produced leading to efficient horizontal gene transfer. 


\section{Introduction}

50 Cholera, caused by the facultative pathogen Vibrio cholerae is far from being extinct. The 51 current Haiti out- break clearly shows that it is a re-emerging disease (Morens et al. 2004).

52 The causative organism $V$. cholerae, apart from being a human pathogen also share an 53 ecological relationship with the host chitinous planktonic copepods during its reside in the 54 aquatic environment (Huq et al. 1983). During their growth on chitin $V$. cholerae can acquire 55 new genetic material from the environment by natural transformation, a mode of Horizontal 56 Gene Transfer (HGT) (Meibom et al. 2005). HGT has been reported as an important 57 phenomenon that contributes in the development of high level of genomic diversity among 58 the Vibrio Spp. (Thompson et al. 2005; Purdy et al. 2005). The transforming foreign DNA maintenance or to the acquisition of new alleles or genes leading to the genetic diversity or evolution (Blokesch et al. 2007). The sero-conversion of $\mathrm{O} 1$ to $\mathrm{O} 139$ by means of natural transformation indicates that HGT may lead to the emergence of new and more virulent strains of $V$. cholerae (Blokesch et al. 2007; Faruque et al. 2003).

64 The major component of the exoskeleton of copepods are chitin, a polymer of $\beta$ - 1, 4-linked 65 N-acetylglucosamine (GlcNAc), has been reported to induce natural competence and transformation in Vibrio Spp. (Meibom et al. 2005). Chitin induces the genes that encode type IV pilus assembly complex. Type IV pilus assembly complex is known to be potentially involved in the DNA uptake process (Meibom et al. 2004; Suckow et al. 2011). The importance of type IV pilus assembly complex in natural competence was determined by disrupting following genes in V. cholerae strain A1552: pilA (VC2423), the gene predicted to encode a type IV pilin; pilQ (VC2630) which encode a homolog of the secretin protein family; and pilB (VC2424) encoding a traffic nucleotide triphosphatase (NTPase), speculated 
74 were tested for natural competence, no transformants were detected compared to the wild 75 type strain.

76 The initiation of competence and transformation occurs via a chitin dependent activation of

77 TfoX, the main regulator of natural transformation (Meibom et al. 2005; Meibom et al.

78 2004). A small RNA (sRNA) $t f o R$ which is transcriptionally induced in presence of

79 (GlcNAc) $)_{2}$ activates the translation of $t f o X$ mRNA (Yamamoto et al. 2011). TfoX regulates

80 the expression of diverse genes including those encoding the proteins that functions in chitin

81 degradation and chitin-induced competence. The three pilus assembly genes pilA, pilB and

82 pilQ, genes encoding four chitinases including ChiA1 and ChiA2, and a chitoporin gene are

83 reported to be induced by TfoX (Meibom et al. 2005).

84 ChiA2, the extracellular chitinase of $V$. cholerae along with the endo-chitinase ChiA1 plays

85 the key role in acquisition of nutrients from the natural environment and helps $V$. cholerae in

86 initialization of chitin utilization pathway (Hunt et al. 2008). The identification of chitin and

87 its oligomers as the inducer of $t f o X$ expression and horizontal gene transfer creates an obvious

88 interest about the role of chitinases in HGT which has not been mentioned or studied by any

89 researcher till date.

90 We show that the environmental condition that supports maximum expression and activity of

91 ChiA2 is also optimum for tfoX expression and HGT. We, by HPLC analyses provide 92 evidence that under the favorable condition $\left(20^{\circ} \mathrm{C}, \mathrm{pH} 5.5\right.$ and $100 \mathrm{mM}$ salt concentration $)$

93 ChiA2 produces significant amount of $(\mathrm{GlcNAc})_{2}$. We hypothesized that under the optimum 94 condition ChiA2 was up-regulated and maximally active to produce significant amount of 95 (GlcNAc $)_{2}$ from chitin. The same environmental condition also induced tfo $X$ expression 96 followed by its translational activation by $(\mathrm{GlcNAc})_{2}$ produced leading to efficient horizontal 97 gene transfer. 
99

100

101

102

103

104

105

106

107

108

109

110

111

112

113

114

115

116

117

123

Materials and Methods

\section{Bacterial culture and growth conditions}

$V$. cholerae $\mathrm{O} 1 \mathrm{El}$ Tor strain $\mathrm{C} 6709$ was used for the studies. A chiA2 mutant strain of $V$. cholerae C6709 was constructed as described previously (Mondal et al. 2014). Wild type $V$. cholerae C6709 and the $\triangle$ chiA2 C6709 was cultured and maintained in tryptic soy broth.

The expression studies of chiA2 and $t f o X$ was done under three different environmental conditions (Table 1). V. cholerae was grown overnight in tryptic soy broth (TSB) medium (BD Difco, Sparks, MD), centrifuged and washed in Phosphate buffered saline (PBS) and $1 \times$ $10^{5}$ cells were inoculated in seawater media $(400 \mathrm{mM} \mathrm{NaCl} ; 10 \mathrm{~mm} \mathrm{KCl} ; 25 \mathrm{mM} \mathrm{MgCl} 2 ; 28$ $\left.\mathrm{mM} \mathrm{MgSO}_{4}\right)$ supplemented with chitin or $(\mathrm{GlcNAc})_{2}$. The inoculated culture then grown separately under the mentioned three different environmental conditions for $24 \mathrm{~h}$.

\section{Isolation of Total RNA}

The total RNA from $V$. cholerae was isolated by using TRIzol reagent (Invitrogen, Carlsbad, CA). This reagent maintained the integrity of RNA during the homogenization of the cells and at the same time it dissolved cellular components of the disrupted cells. Briefly, the cells were homogenized thoroughly with TRIzol and incubated at room temperature for at least 5 mins for complete dissociation of the nucleoprotein complexes. Chloroform $\left(1 / 5^{\text {th }}\right.$ volume of TRIzol) was added to the homogenate and shaken vigorously for $15 \mathrm{~s}$, incubated 2-3 mins at room temperature; centrifuged at $12000 \times g$ for 15 mins at $4{ }^{\circ} \mathrm{C}$. The aqueous phase was transferred to a fresh tube and isopropanol $\left(1 / 2^{\text {nd }}\right.$ volume of TRIzol) was added, incubated at room temperature for 10 mins and centrifuged at $12000 \times g$ for 10 mins at $4^{\circ} \mathrm{C}$. the pellet was washed with $75 \%$ ethanol, air dried and dissolved in desired volume of nuclease free water. The isolated RNA was stored at $-70{ }^{\circ} \mathrm{C}$. 


\section{Quantitative PCR}

126 The expression of $t f o X$ and chiA2 was studied quantitatively by Quantitative PCR (qPCR) 127 using Power SYBR Green PCR master Mix (Applied Biosystems, Foster City, California) 128 and a set of primers (Table 2) in a 7500 Real Time PCR detection system (Applied 129 Biosystems, Foster City, California). V. cholerae or $\Delta$ chiA2 V. cholerae was grown for $24 \mathrm{~h}$ 130 in sea-water media supplemented with chitin separately under the three different environmental conditions as mentioned in Table 1. The total RNA isolated from $V$. cholerae was converted to cDNA using Reverse Transcription System (Promega, Madison, WI) and was used as template. The recA gene was used as internal control. Comparative RNA expression was studied by comparing the $\Delta \Delta \mathrm{Ct}$ values determined from the qPCR (Livak et al., 2001). To study the effect of (GlcNAc) $)_{2}$ in tfoX expression in $\Delta c h i A 2$ mutants the mentioned experiment was performed in a similar manner in sea-water media supplemented with $6 \mathrm{mM}(\mathrm{GlcNAc})_{2}$ and the expression of $t f_{0} X$ was measured by qPCR. For the qPCR reactions $\operatorname{rec} A$ was used as internal control.

\section{Chitinase activity assay}

141 The N-acetylglucosamine concentration in the reaction mixture and the chitinase activity were determined by previously established Di-nitrosalicylic acid (DNS) method (Miller et al. 143 1959). The experiment was performed as described in Mondal et al. 2014. The amount of 144 reducing sugar was then estimated spectrophotometrically. The specific activity of the 145 enzyme was calculated by measuring the amount of GlcNAc produced in $\mu \mathrm{mole} / \mathrm{mg}$ of $146 \mathrm{protein} / \mathrm{min}$. The velocity of each reaction was calculated by measuring the amount of 147 GlcNAc produced in $\mu$ mole/ $\mathrm{ml}$ of reaction mixture/min. The Michaelis Menten constant $\mathrm{K}_{\mathrm{m}}$ 
and the maximum velocity $\mathrm{V}_{\max }$ were calculated from a Lineweaver Burk plot. The catalytic constant $\mathrm{K}_{\text {cat }}$ and the catalytic efficiency $\left(\mathrm{K}_{\mathrm{m}} / \mathrm{K}_{\text {cat }}\right)$ were also determined.

\section{Generation of $V$. cholerae growth curve}

The variation in $V$. cholerae growth under the three different environmental conditions (Table 1) was studied by growth curve analyses. Overnight cultures of the wild type $V$. cholerae and $\triangle$ chiA2 V. cholerae were diluted separately in sea-water media (pH 8.0, salinity $300 \mathrm{mM})$ supplemented with of $2 \%$ chitin. The cultures were grown for $72 \mathrm{~h}$ with shaking. The viable cells were enumerated after $4 \mathrm{~h}, 8 \mathrm{~h}, 10 \mathrm{~h}, 12 \mathrm{~h}, 24 \mathrm{~h}, 30 \mathrm{~h}, 48 \mathrm{~h}$ and $72 \mathrm{~h}$ time interval by plating the cultures on thiosulfate-citrate-bile-salts-sucrose (TCBS) agar plate.

\section{Effect of environmental conditions on $V$. cholerae $\mathrm{HGT}$}

The $V$. cholerae HGT was performed by following a previously described method (Meibom et al. 2005) with a little modification. In brief, $1 \times 10^{9} \mathrm{CFU}$ of overnight cultures of $V$. cholerae and $\triangle$ chiA2 V. cholerae were inoculated with sea-water media supplemented with chitin and the transformation reaction was performed under three different environmental conditions (Table 1) with $3 \mu \mathrm{g} / \mathrm{ml}$ transforming ampicillin resistant GFP plasmid. The cultures were then incubated for $18 \mathrm{~h}$ in static condition. After $18 \mathrm{~h}$ the cultures were plated onto ampicillin containing LB plates.

\section{HPLC analyses of the end products of ChiA2 treated mucin}

HPLC analyses were done to estimate oligosaccharides produced as an end product of chitin after ChiA2 treatment. The oligosaccharides were eluted from the column by using a gradient of $60-80 \%$ acetonitrile and water. The retention time of each oligosaccharide was compared with commercially available standards. In detail, HPLC analyses were done using 
173 a $4.6 \mathrm{~nm} \times 250,5 \mu \mathrm{m}$ Zorbax Carbohydrate Analysis Column (Agilent Technologies, Santa

174 Clara, CA) connected to a Shimadzu Prominence 20A, HPLC system. LC solutions 175 (Shimatzu, Kyoto, Japan) program was used to control, calibrate and analyze the HPLC data. 176 In $20 \mathrm{mM}$ phosphate buffer $\mathrm{pH} 7.4,1 \mathrm{mg} / \mathrm{ml}$ of crab shell chitin (Sigma, St. Louis, MO) was 177 incubated with $50 \mu \mathrm{g} / \mathrm{ml}$ purified ChiA2 at $30{ }^{\circ} \mathrm{C}$ for $3 \mathrm{~h}$. The reaction was terminated by $17810 \%$ trichloroacetic acid (HIMEDIA, Mumbai, India) (v/v). Eighty microliter of the 179 terminated reaction mixtures were injected to the HPLC Column operated at $40{ }^{\circ} \mathrm{C}$. The 180 oligosaccharides were eluted from the column by using a gradient of $60-80 \%$ acetonitrile and water. The eluted fraction was passed immediately by LC PDA detection system to detect the components of the eluted fraction. The retention time of each oligosaccharide was compared with commercially available standards.

\section{Statistical analysis}

All the experiments were repeated at least three times. Data are expressed as means \pm standard error (SE). One way ANOVA was used to analyze the data. A $P$ value of $<0.05$ was considered statistically significant.

\section{Results}

\section{Effect of environmental conditions on $c h i A 2$ and $t f o X$ expression}

192 The expression of chiA2 was analyzed in C6709 strains, under three different conditions mentioned in Table 1. The conditions were selected depending on the variation of growth of $V$. cholerae N16961 and the expression of chiA2 over a range of temperature, $\mathrm{pH}$ and salt concentration (supplementary material Fig S1 to S3). analysis of the expression showed, under condition I the expression of chiA2 was 3.6-fold 
198

199

200

201

202

203

204

205

206

207

208

209

210

211

212

213

214

215

216

217

higher compared to the expression under condition II. The expression of chiA2 under condition III was lowest and was 5.9- fold less compared to the expression under condition I. This result indicated that the most favorable condition for the $V$. cholerae C6709 chiA2 expression was condition I that is $20^{\circ} \mathrm{C}$, pH 5.5 and $100 \mathrm{mM}$ salt concentration. This observation also showed that the optimum condition for $V$. cholerae chiA2 expression varies with $V$. cholerae strain.

Analyses of the $t f o X$ expression of $V$. cholerae strain $\mathrm{C} 6709$ under the three mentioned conditions showed condition I as the most favorable condition for $t f o X$ expression (Fig 1B). Under condition I the $t f_{0} X$ expression was 4.5-fold higher compared to the expression under condition II. Under condition III $t f o X$ expression was the lowest.

These results indicated that condition $\mathrm{I}$ is the most favorable condition for chiA2 expression as well as $t f o X$ expression.

\section{Effect of environmental factors on activity of ChiA2}

Activity of pure ChiA2 was studied under the mentioned three environmental conditions (Table I). The variations in ChiA2 activity under the different environmental conditions were studied by analyzing ChiA2 specific activity, using chitin as assay substrate. Condition I was found as the most favorable condition for $V$. cholerae C6709 ChiA2 activity compared to the rest two conditions (Fig 2). Under condition II also ChiA2 showed significant activity. Comparison of the specific activities of ChiA2 showed that it was 4.1-fold more active under condition I compared to condition III. Under condition II ChiA2 showed 3.3-fold more activity compared to condition III. Condition III was found as the most unfavorable condition for C6709 ChiA2 activity. This data showed that environmental condition I was most favorable for $V$. cholerae C6709 ChiA2 activity. 


\section{$V$. cholerae requires an active $\mathrm{ChiA2}$ as well as optimum environmental condition for a} successful growth

The growth of $V$. cholerae was studied under the previously mentioned three different conditions (Table1). The $\Delta c h i A 2 V$. cholerae strain showed poor growth under the all three conditions. At $48 \mathrm{~h}$ the wild type $V$. cholerae showed $\sim 100$-fold higher growth under condition I compared to the $\triangle$ chiA2 $V$. cholerae (Fig 3A). The growth of wild type $V$. cholerae was $\sim 10$-fold decreased under condition II compared to condition I (Fig 3B). Under condition III both wild type and $\triangle$ chiA $2 \mathrm{~V}$. cholerae did not show significant growth and it was almost 1000-fold less compared to condition I (Fig 3C). These observations indicated that condition I was the most favorable condition for $V$. cholerae C6709 growth and ChiA2 is essential for the growth. In absence of chiA2, $V$. cholerae growth was compromised even under the favorable conditions. On the other hand it is also observed that even if chiA2 is present $V$. cholerae shows poor growth under the unfavorable conditions.

We observed $\triangle$ chiA2 $V$. cholerae was able to retrieve the wild type growth when the growth media was supplemented with $(\mathrm{GlcNAc})_{2}$. We observed that under condition I, $\Delta$ chiA2 $\mathrm{V}$. cholerae showed similar growth with respect to wild type, in (GlcNAc) $)_{2}$ supplemented growth media (Fig 3A). Under condition I the $\triangle$ chiA2 V. cholerae growth increased by $100-$ fold in presence of (GlcNAc $)_{2}$ compared to the non-(GlcNAc $)_{2}$ supplemented growth media. Under condition II, the similar change in $\triangle$ chiA2 V. cholerae growth was observed (Fig 3B). Condition III was not favorable for the growth of $V$. cholerae (Fig 3C).

\section{Effect of environmental condition on $V$. cholerae HGT}

In the previous studies we found condition I among the three mentioned conditions (Table 1), as the most favorable for chiA2 and $t f o X$ expression as well as ChiA2 activity. $V$. cholerae HGT under the defined three environmental conditions showed that condition I was the most 
248 favorable condition for $V$. cholerae HGT (Fig 4). Under condition I the number of 249 transformants calculated was $\sim 6.7$ to 8.5 - fold higher for wild type $V$. cholerae compared to 250 the other two conditions. However, the $\triangle$ chiA2 V. cholerae showed reduced level of natural 251 competence and less number of transformants compared to the wild type strain irrespective of 252 the condition selected. Under condition I, the number of transformants of $\Delta$ chiA2 V. cholerae 253 increased up to 13.5 - fold when $(\mathrm{GlcNAc})_{2}$ was supplemented compared to the number of 254 transformants of $\triangle$ chiA2 $V$. cholerae without (GlcNAc) $)_{2}$ supplement in the growth media. 255 This observation indicated that in presence of $(\mathrm{GlcNAc})_{2}$ the $\Delta$ chiA2 V. cholerae was capable 256 of HGT and among the three environmental conditions condition I is the most favorable for $257 \quad V$. cholerae HGT.

\section{ChiA2 releases significant amount of GlcNAc dimer from Chitin}

The end products of ChiA2-treated chitin were analyzed by HPLC and the components were identified with the corresponding standards. In Fig 5A, the chromatogram showed four distinct peaks. Peak 1 with retention time 5.5 min corresponded to the peak of GlcNAc (Fig 5B), the main product released from chitin on ChiA 2 treatment. It occupied $35 \%$ of the total peak area. Peak $2 \mathrm{~A}$ with retention time $6.2 \mathrm{~min}$ and peak $2 \mathrm{~B}$ with retention time $6.5 \mathrm{~min}$ eluted in close proximity. Peak 2B with retention time $6.5 \mathrm{~min}$ (Fig 5A) corresponded to $\alpha$ $(\text { GlcNAc })_{2}$ (Fig 2B). Peak 2A did not have any standards and we presumed it with all possibilities as the peak of $\beta-(\mathrm{GlcNAc})_{2}$. Peak $2 \mathrm{~A}$ occupied $22.2 \%$ of the total peak area and peak 2B occupied $18.2 \%$ of the total peak area (Table 3). Peak III with retention time 7.42 (Fig 5A) corresponded to the peak of (GlcNAc) $)_{3}$ (Fig 5B) and it occupied only $9.6 \%$ of the total peak area (Table 3). So this data evidenced that chitin on treatment with ChiA2 releases significant amount of both the enantiomers of $(\mathrm{GlcNAc})_{2}$ besides the release of its main 
272 product GlcNAc. The presence of small peak of $(\mathrm{GlcNAc})_{3}$ in the chromatogram was might

273 be due to the un-degraded (GlcNAc) 3.

\section{Discussion}

276 In this study we demonstrated the importance of the extracellular chitinase ChiA2 in $V$. 277 cholerae natural transformation, a mode of HGT. ChiA2 is important for initialization of $V$. 278 cholerae chitin utilization pathway. It helps to degrade the insoluble chitinous exo-skeleton 279 of copepods into its soluble monomers and oligomers which then be readily utilized by $V$. 280 cholerae (Hunt et al. 2008). Chitin acts as an inducer of $V$. cholerae natural transformation 281 and facilitates the uptake of new genetic material from the environment. Cholera is a climate 282 related infectious disease (Lipp et al. 2002). Abiotic environmental factors regulate the 283 survival and the association of $V$. cholerae with the zooplanktons via the regulation of expression and activity of chitinase genes (Bhowmick et al. 2007; Lipp et al. 2002). The most favorable conditions for ChiA2 expression and activity of $V$. cholerae $\mathrm{O} 1 \mathrm{El}$ Tor strain C6709 was observed at temperature $20^{\circ} \mathrm{C}, \mathrm{pH} 5.5$ and $100 \mathrm{mM}$ salt concentration. $V$. 287 cholerae N16961 ChiA2 expression and activity works favorably under condition II 288 (Supplementary material, Fig S1 to S5). However, this strain is a natural mutant of hapR and did not undergo natural transformation. So we used the hapR positive $V$. cholerae O1 El Tor strain C6709 for our study. Different environmental conditions were found as favorable for chiA2 expression of two different strains of $V$. cholerae $\mathrm{O} 1 \mathrm{El}$ Tor.

292 As reported earlier, the chitinase genes of $V$. cholerae O1 El Tor mostly shows high level of 293 expression and activity at low temperature (Bhowmick et al. 2007). Temperature around 25 $294{ }^{\circ} \mathrm{C}$ has been reported to increase $V$. cholerae counts in aquatic reservoirs and increased chitinolytic activities (Louis et al. 2003). We found $V$. cholerae C6709 ChiA2 was maximally 
297

298

299

300

301

302

303

acidic pH (Annamalai et al, 2010; Dahiya et al, 2005; Murao et al, 1992; Ohtakara et al. 1979). V. cholerae C6709 ChiA2 was also observed to be active at $\mathrm{pH} 5.5$ having a similarity with the chitinase of Enterobacter spp. NRG4 (Dahiya et al. 2005).

We observed that the expression of $t f o X$, the central regulator of $V$. cholerae HGT, was maximum under the same environmental conditions which were found optimum for ChiA2 expression and activity indicating regulation by the same environmental conditions.

The most favorable condition for $V$. cholerae C6709 growth was exactly the same that supports the expression of chiA2 and tfoX. However, the $\Delta$ chiA2 V. cholerae did not show significant growth under this condition indicating that ChiA2 is essential for $V$. cholerae C6709 survival and growth in aquatic environment during its reside on the chitinous surface of copepods. This data corroborated with the data of Meibom et al. (2004) in which it has been reported that a $\triangle$ chiA2 $V$. cholerae of another O1 El Tor strain N16961 also exhibited reduced growth compared to the wild type (Meibom et al. 2004). This indicated that ChiA2 is important for survival of $V$. cholerae irrespective of the strains. However, we found that the $\triangle$ chiA2 V. cholerae C6709 strain could retrieve wild type growth phenotype when the growth media was supplemented with (GlcNAc) $)_{2}$. This observation suggested that $(\mathrm{GlcNAc})_{2}$ might be an important nutrient required for growth of $V$. cholerae C6709. Uchiyama et al. in year 2003 reported that in Serratia marcescens the expression of chitinases and chitin binding proteins are induced by (GlcNAc) $)_{2}$ (Uchiyama et al. 2003). The $S$. marcescens mutants that are defective in $(\mathrm{GlcNAc})_{2}$ transport are unable to express the chitinases (Uchiyama et al. 2003). These reports suggest that (GlcNAc) $)_{2}$ has an important role in induction of chitinase expression that are essential for nutrient supply to the bacteria via the chitin degradation pathway in the aquatic environment. Further detailed explanation of this phenomenon was given by a study that reported (GlcNAc) $)_{2}$ activates translation of tfoX (Yamamoto et al. 
321 2011), that regulates the expression of the genes those involved in chitin degradation and 322 natural competence (Meibom et al. 2005).

323 Our results on the effect of environmental conditions on $V$. cholerae HGT demonstrated that 324 conditions favoring the expression of chiA2 and tfoX and the activity of ChiA2, was also 325 optimum for $V$. cholerae HGT. However, dysregulation of ChiA2 under the optimum 326 condition showed reduced natural competence compared to the wild type strain. Addition of 327 exogenous $(\mathrm{GlcNAc})_{2}$ supported the role of ChiA2 in HGT. This result clearly states that 328 ChiA2 acts on chitin to release $(\mathrm{GlcNAc})_{2}$ that supports $V$. cholerae HGT. Reported that, in presence of $(\mathrm{GlcNAc})_{2}$ translational activation of $t f o X$ occurs via the transcriptional activation of $t f o R$ small RNA. Activated $t f o X$, the regulator of HGT then up-regulates the expression of genes involved in natural competence (Yamamoto et al. 2011).

Taken together we observed that the maximum expression of chiA2 and $t f o X$ is optimum under the same environmental conditions that also favor the chitinolytic activity, $V$. cholerae growth as well as $V$. cholerae HGT. We speculated that, under the favorable environmental conditions, ChiA2 was up-regulated and maximally active to produce significant amount of $(\mathrm{GlcNAc})_{2}$ from chitin. Presence of significant amount of $(\mathrm{GlcNAc})_{2}$ in the end product of ChiA2 treated chitin provides a strong evidence about this phenomenon. The $t f o R$ small RNA regulator is transcriptionally induced in presence of $(\mathrm{GlcNAc})_{2}$ and activates the translation of tfoX mRNA (Yamamoto et al, 2011). Activated TfoX then regulates the expression of genes involved in chitin induced competence (Meibom et al. 2005). The same environmental conditions also induced $t f o X$ expression followed by its translational activation by (GlcNAc) $)_{2}$ produced leading to efficient HGT.

\section{Acknowledgement}


346 The authors are grateful to the Indian Council of Medical Research, internal to our 347 organization for the financial support. The funders had no role in study design, data collection 348 and analysis, decision to publish, or preparation of the manuscript. Moumita Mondal has been 349 supported by the funds from University Grants Commission [UGC sanction No. F.2.77/98 $350 \quad(\mathrm{SA}-\mathrm{I})]$

351

352

353

354

355

356

357

358

359

360

361

362

363

364

365

366

367

368

369

370

371

372

373

374

375

376

377

378

379

\section{References}

Annamalai N., Giji S., Arumugm M., Balasubramanium T. (2010). Purification and characterization of Chitinase from Micrococcus sp. AG84 isolated from marine environment. Afr. J. Microbial. Res; 4(24). 2822-28.

Bhowmick R., Ghosal A., Chatterjee N. S. (2007). Effect of environmental factors on expression and activity of chitinase genes of vibrios with special reference to Vibrio cholerae. Journal of Applied Microbiol 103. 97-108.

Blokesch M., Schoolnik G. K. (2007). Serogroup Conversion of Vibrio cholerae in Aquatic Reservoirs. PLoS Pathog 3: e81. doi:10.1371/journal.ppat.0030081.

Dahiya N., Tewari R., Tiwari R. P., Hoondal G. (2005). Production of an antifungal chitinase from Enterobacter sp.NRG4 and its application in protoplast production. World. J. Microbiol. Biotechnol., 21: $1611-1616$.

Faruque S. M., Mekalanos J. J. (2003). Pathogenicity islands and phages in Vibrio cholerae evolution. Trends Microbiol 11: 505-510.

Huq A., Small E. B., Colwell R. R. et al. (1983) Ecological relationships between Vibrio cholerae and planktonic crustacean copepods. Appl. Environ. Microbiol., 45:275-283

Hunt D. E., Gevers D. et al. (2008). Conservation of chitin utilization pathway in Vibrionaceae. Appl. Environ. Microbiol. 74 (1): 44-51

Livak K. J., Schmittgen T. D. (2001). Analysis of relative gene expression data using real-time quantitative OCR and the $2^{-\Delta \Delta C}$ Tethod. Methods. 25, 402- 408. 
Louis V. R., Russek-Cohen E., Choopun N., Rivera I. N. G., Gangle B., Jiang S. C., Rubin A., Patz J.

A., Huq A., Colwell R. R. (2003) Predictability of Vibrio cholerae in Chesapeake Bay. Appl Environ

Microbiol 69:2773-2785

384

Meibom K. L., Li X. B., Nielsen A. T., Wu C. Y., Roseman S., et al. (2004). The Vibrio cholerae chitin utilization program. Proc Natl Acad Sci USA 101: 2524-2529.

Meibom K. L., Blokesch M., Dolganov N. A., Wu C-Y., Schoolnik G. K. (2005). Chitin induces natural competence in Vibrio cholerae. Science 310: 1824-1827.

390

Miller G. L. (1959). Use of Di nitro salicylic acid reagent for determination of reducing

393

Morens D. M., Folkers G. K., Fauci A. S. (2004). The challenge of emerging and re- emerging infectious diseases. Nature 430: 242-249.

396

Murao S., Kawada T., Itoh H., Oyama H., Shin T. (1992). Purification and characterization of a novel type of chitinase from Vibrio alginolyticus TK-22. Biosci. Biotechnol. Biochem., 56: 368-369.

402

Ohtakara A., Mitsutomi M., Uchida Y. (1979). Purification and some properties of chitinase from Vibrio sp., J. Ferment. Technol., 57: 169-173.

405

Purdy A., Rohwer F., Edwards R., Azam F., Bartlett D. H. (2005). A glimpse into the expanded genome content of Vibrio cholerae through identification of genes present in environmental strains.

409

410

Suckow G., Seitz P., Blokesch M. (2011). Quorum Sensing Contributes to Natural Transformation of 412

413 Thompson J. R., Pacocha S., Pharino C., Klepac-Ceraj V., Hunt D. E. et al. (2005). Genotypic 414 Diversity Within a Natural Coastal Bacterioplankton Population. Science 307, 1311 (2005). 
415

416 Yamamoto S., Izumiya H., Mitobe J., Morita M., Arakawa E., et al. (2011). Identification of a Chitin417 Induced Small RNA That Regulates Translation of the tfoX Gene, Encoding a Positive Regulator of 418 Natural Competence in Vibrio cholerae. J Bacteriol 193: 1953-1965.

419

420

421

422

423

424

425

426

427

428

429

430

431

432

433

434

435

436

437

438

439

440

441

442

443

444

445

446

447

448

449

450

451

452

453

454

455

456

457

458

459

460

461

462

463 
464

465

466

467

\begin{tabular}{|l|l|l|l|l|}
\hline Conditions & Temperature & $\mathrm{pH}$ & $\begin{array}{l}\text { Salinity (mM } \\
\text { sodium } \\
\text { chloride) }\end{array}$ & Media used \\
\hline Condition I & $20^{\circ} \mathrm{C}$ & 5.5 & 100 & $\begin{array}{l}\text { Sea-water } \\
\text { media } \\
\text { supplemented } \\
\text { with } 0.8 \% \\
\text { chitin }\end{array}$ \\
\hline Condition II & $30^{\circ} \mathrm{C}$ & 8.0 & 300 & $\begin{array}{l}\text { Sea-water } \\
\text { media } \\
\text { supplemented } \\
\text { with 0.8\% } \\
\text { chitin }\end{array}$ \\
\hline Condition III & $42^{\circ} \mathrm{C}$ & & 600 & $\begin{array}{l}\text { Sea-water } \\
\text { media } \\
\text { supplemented } \\
\text { with } 0.8 \% \\
\text { chitin }\end{array}$ \\
\hline
\end{tabular}

Table 1: the definition of the environmental conditions selected for the study
468

469

470

471

472

473

474

475

476

477

478

479

480

481

482

483

484

485

486

487

488

489

490

491

492

493

494

495 
496

497

498

499

500

501

502

503

504

505

506

507

508

509

510

511

512

513

514

515

516

517

518

519

520

521

522

523

524

525

526

527

528

529

530

531

532

533

Table 2: List of primers

\begin{tabular}{|l|l|}
\hline Primers & Sequence \\
\hline $\begin{array}{l}\text { chiA2 real time } \\
\text { primer F }\end{array}$ & $5^{\prime}$ ACAACGACACCGCCAGTAAAC 3' \\
\hline $\begin{array}{l}\text { chiA2 real time } \\
\text { primer R }\end{array}$ & $5^{\prime}$ TGCCCCAGTCAAGGTCACAGC \\
\hline chiA2XbaI A F & $5^{\prime}$-CAGCTCTAGACTCTGATACTGACGTTCGAG-3' \\
\hline chiA2 B R & $\begin{array}{l}5^{\prime} \text {-CCCATCCACTATAAACTAACACAATGGCTTGAGGTGTGAAC- } \\
3^{\prime}\end{array}$ \\
\hline chiA2 C F & $\begin{array}{l}5^{\prime} \text {-TGTTAGTTTATAGTGGATGGGGGAACAGGTGGTGATGCTT- } \\
3^{\prime}\end{array}$ \\
\hline ChiA2 SACI D & $5^{\prime}$-GAATCGAGCTCGATTGCATTCCCAATGAG-3' \\
\hline
\end{tabular}

2




\begin{tabular}{|c|c|c|c|c|c|c|}
\hline & & & \multicolumn{2}{|c|}{ Standards } & \multicolumn{2}{|c|}{$\begin{array}{l}\text { ChiA2-chitin } \\
\text { reaction }\end{array}$} \\
\hline $\begin{array}{c}\text { Peak } \\
\text { number }\end{array}$ & $\begin{array}{l}\text { Sub } \\
\text { peak }\end{array}$ & $\begin{array}{c}\text { Peak } \\
\text { Identity }\end{array}$ & $\begin{array}{r}\text { Ret. Time } \\
\text { (mins) }\end{array}$ & $\begin{array}{c}\% \\
\text { Area }\end{array}$ & $\begin{array}{c}\text { Ret. Time } \\
\text { (mins) }\end{array}$ & $\%$ Area \\
\hline 1. & & (GlcNAc) & $5.5 \pm 0.05$ & 35.8 & $5.56 \pm 0.05$ & 35.09 \\
\hline \multirow[b]{2}{*}{2} & $\bar{A}$ & $(\mathrm{GlcNAc})_{2} \beta$ & NO* & \multirow{2}{*}{19.7} & $6.20 \pm 0.03$ & 22.23 \\
\hline & $\mathrm{B}$ & $(\text { GlcNAc })_{2} \alpha$ & $6.6 \pm 0.03$ & & $6.57 \pm 0.03$ & 18.21 \\
\hline 3. & & $(\mathrm{GlcNAc})_{3}$ & $7.5 \pm 0.02$ & 16.1 & $7.42 \pm 0.02$ & 9.66 \\
\hline 4. & & $(\text { GlcNAc })_{6}$ & $11.15 \pm 0.03$ & 6.5 & $\mathrm{NO}^{*}$ & - \\
\hline
\end{tabular}

Table 3: HPLC analysis of the end products of ChiA2 treated chitin 


\section{$571 \quad$ Figure legends}

572

573

574

Figure 1. A. Graphical representation of the effect of environmental conditions on chiA2 expression. Effect of environmental conditions on chiA2 expression was studied under the three mentioned conditions (Table 1). V. cholerae cells were cultured in sea water media supplemented with chitin separately under the conditions mentioned in Table 1. After $24 \mathrm{~h}$ growth the total RNA was isolated and chiA2 expression was analyzed by quantitative PCR.

578 Maximum expression was observed under condition I which was 3.6-fold higher compared to the expression under condition II. Lowest expression was observed under condition III. B. Graphical representation of effect of environmental factors on $t f o X$ expression. Under varying environmental conditions the expression of $t f o X$ was studied following the similar method used to study the chiA2 expression. Quantitative analyses of $t f o X$ expression by qPCR showed condition I is the most favorable for $t f o X$ expression and condition III least favorable for $t f o X$ expression. Under condition I the tfox expression was 4.5- fold higher compared to the expression under condition II. All the experiment was carried out thrice and the data represented as mean $\pm \mathrm{SEM}$. cholerae C6709 ChiA2 showed maximum activity and under condition III the activity was minimum while, under condition II ChiA2 showed moderate activity. Under condition I the activity of ChiA2 was 4.3 -fold higher compared to the activity under condition III. Under 
596 activity under condition I. The experiment was performed thrice and the data presented here 597 as mean $\pm \mathrm{SEM}$.

Figure 3. Graphical representation of the effect of environmental conditions on $V$. cholerae C6709 growth. Effect of environmental conditions on V. cholerae C6709 growth was studied in sea water media supplemented with $2 \%$ chitin separately under the mentioned three environmental conditions (Table 1). The growth curves were generated by plotting the number of viable cells $(\mathrm{CFU} / \mathrm{ml})$ in $\mathrm{Y}$-axis again the time interval plotted in $\mathrm{X}$ - axis. The wild type $V$. cholerae C6709 was cultured in sea water media supplemented with $2 \%$ chitin separately under the three mentioned environmental conditions (Table 1). The number of viable cells after the time intervals ( 0 h, 2 h, 4 h, 8 h, 10 h, 12 h, 24 h, 30 h, $48 \mathrm{~h}$ and 72 h) viable cells after the time intervals $(0 \mathrm{~h}, 2 \mathrm{~h}, 4 \mathrm{~h}, 8 \mathrm{~h}, 10 \mathrm{~h}, 12 \mathrm{~h}, 30 \mathrm{~h}, 48 \mathrm{~h}$ and $72 \mathrm{~h}$ ) was enumerated by plate count method using TCBS plates. Figure presents the growth curve of $V$. cholerae C6709 wild type and the $\triangle$ chiA2 strains under A. condition I, B. condition II, C. condition III. overnight cultures of $V$. cholerae and $\triangle$ chiA2 $V$. cholerae were inoculated with sea-water media and the transformation reaction was performed under three different environmental conditions (Table 1) with $3 \mu \mathrm{g} / \mathrm{ml}$ transforming ampicillin resistant GFP plasmid. The graph 
621 shows that condition I is the most favorable for $V$. cholerae HGT compared to the other two 622 conditions. However, the $\triangle$ chiA2 V. cholerae did not show HGT under any of the conditions 623 except in presence of (GlcNAc) $)_{2}$. The experiment was performed thrice and the data 624 presented as mean $\pm \mathrm{SEM}$.

Figure 5. HPLC analysis of end products of ChiA2-treated chitin. The chitin-ChiA2 628 Reactions were carried as mentioned in the materials and methods section. The end products were analyzed using a $4.6 \mathrm{~nm} \times 250,5 \mu \mathrm{m}$ Zorbax carbohydrate analysis column (Agilent indicates presence of GlcNAc. Peak 2 indicates presence of $(\mathrm{GlcNAc})_{2}$ which is divided into two separate peaks: peak 2A and peak 2B. Peak 2A with retention time 6.2 minutes probably is for the $\beta$ enantiomer and peak $2 \mathrm{~B}$ with retention time 6.5 minutes is for a enantiomer of $(\mathrm{GlcNAc})_{2}$. Peak 3 with retention time 7.4 minutes indicates the presence of $(\mathrm{GlcNAc})_{3}$. B.

637 This is the HPLC chromatogram of standards of GlcNAc, (GlcNAc) $)_{2}$, (GlcNAc) $)_{3}$ and $638(\mathrm{GlcNAc})_{6}$. The chromatogram shows 4 distinct peaks. Peak 1 with retention time 5.5 639 minutes is for GlcNAc. Peak 2 with retention time 6.6 minutes is for a-(GlcNAc) $)_{2}$. Peak 3 640 with retention time 7.5 minutes is for $(\mathrm{GlcNAc})_{3}$ and peak 4 with retention time 11.1 minutes 641 is for $(\mathrm{GlcNAc})_{6}$. 

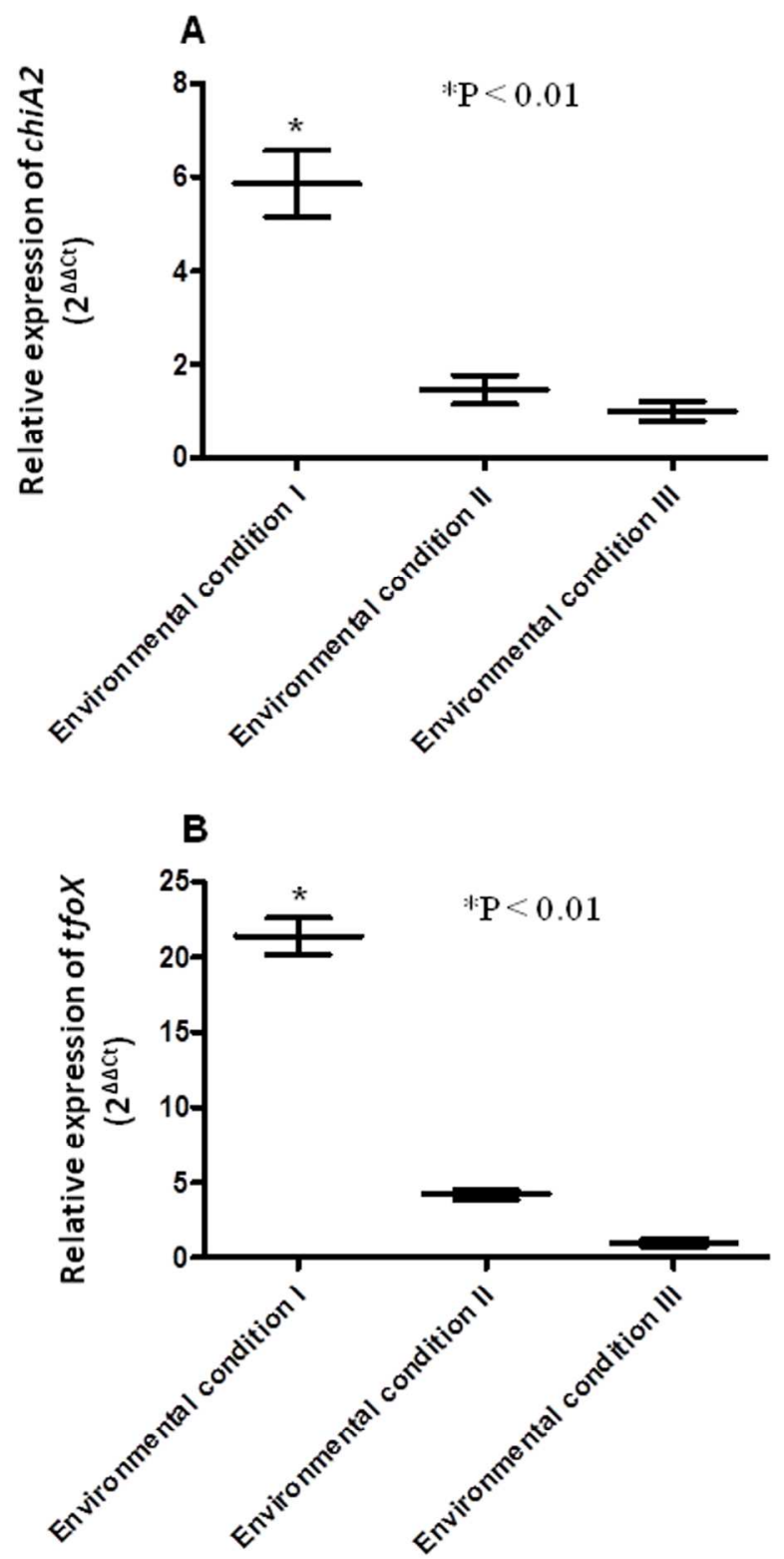

Figure 1, Mondal et al, 2015

Figure 1. A. Graphical representation of the effect of environmental conditions on chiA2 expression. Effect of environmental conditions on chiA2 expression was studied under the three mentioned conditions (Table 1 ). $\mathrm{V}$. cholerae cells were cultured in sea water media supplemented with chitin separately under the conditions mentioned in Table 1 . After $24 \mathrm{~h}$ growth the total RNA was isolated and chiA2 expression was analyzed by quantitative PCR. Maximum expression was observed under condition I which was 3.6-fold higher compared

to the expression under condition II. Lowest expression was observed under condition III. B. Graphical representation of effect of environmental factors on tfoX expression. Under varying environmental conditions the expression of tfoX was studied following the similar method used to study the chiA2 expression. Quantitative analyses of tfoX expression by qPCR showed condition I is the most favorable for tfoX expression and condition III least favorable for tfoX expression. Under condition I the tfox expression was 4.5- fold higher compared to the expression under condition II. All the experiment was carried out thrice and the data represented as mean \pm SEM. $156 \times 210 \mathrm{~mm}(300 \times 300 \mathrm{DPI})$ 


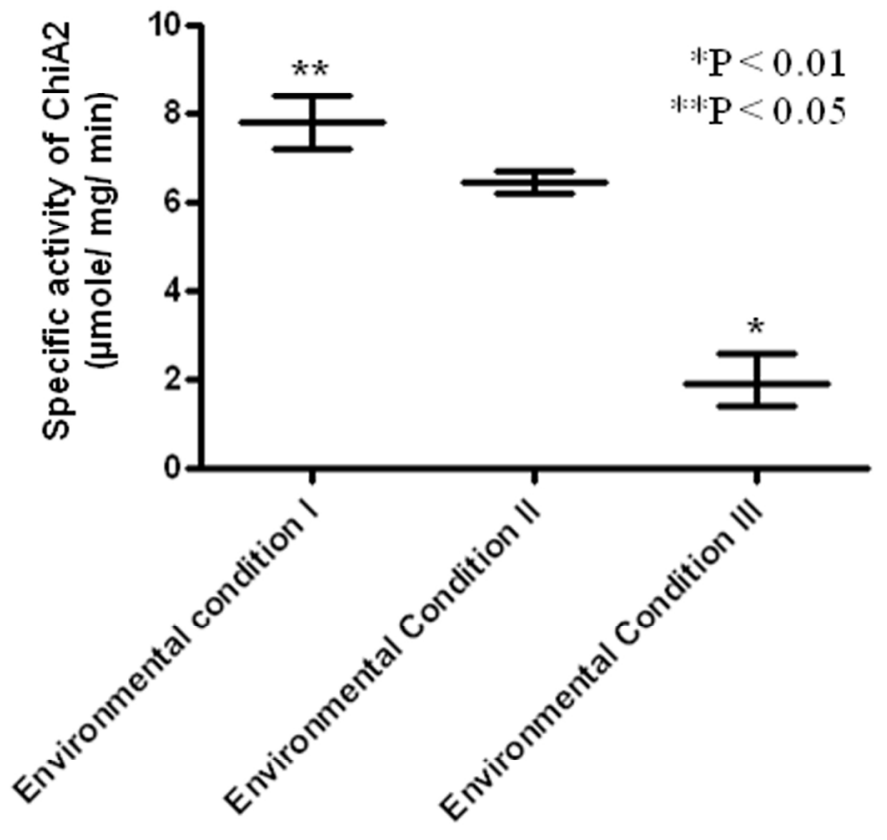

Figure 2, Mondal etal, 2015

Figure 2. Graphical representation of the effect of environmental conditions on ChiA2 activity. Activity of V. cholerae C6709 ChiA2 was studied under the varying environmental conditions. Activity assay of ChiA2 was performed separately under the mentioned environmental conditions (Table I) using $2 \%$ chitin as assay substrate. Under condition I V. cholerae C6709 ChiA2 showed maximum activity and under condition III the activity was minimum while, under condition II ChiA2 showed moderate activity. Under condition I the activity of ChiA2 was 4.3-fold higher compared to the activity under condition III. Under condition II ChiA2 showed significant activity but it was 1.2- fold less compared to the activity under condition I. The experiment was performed thrice and the data presented here as mean \pm SEM. $155 \times 111 \mathrm{~mm}(300 \times 300 \mathrm{DPI})$ 

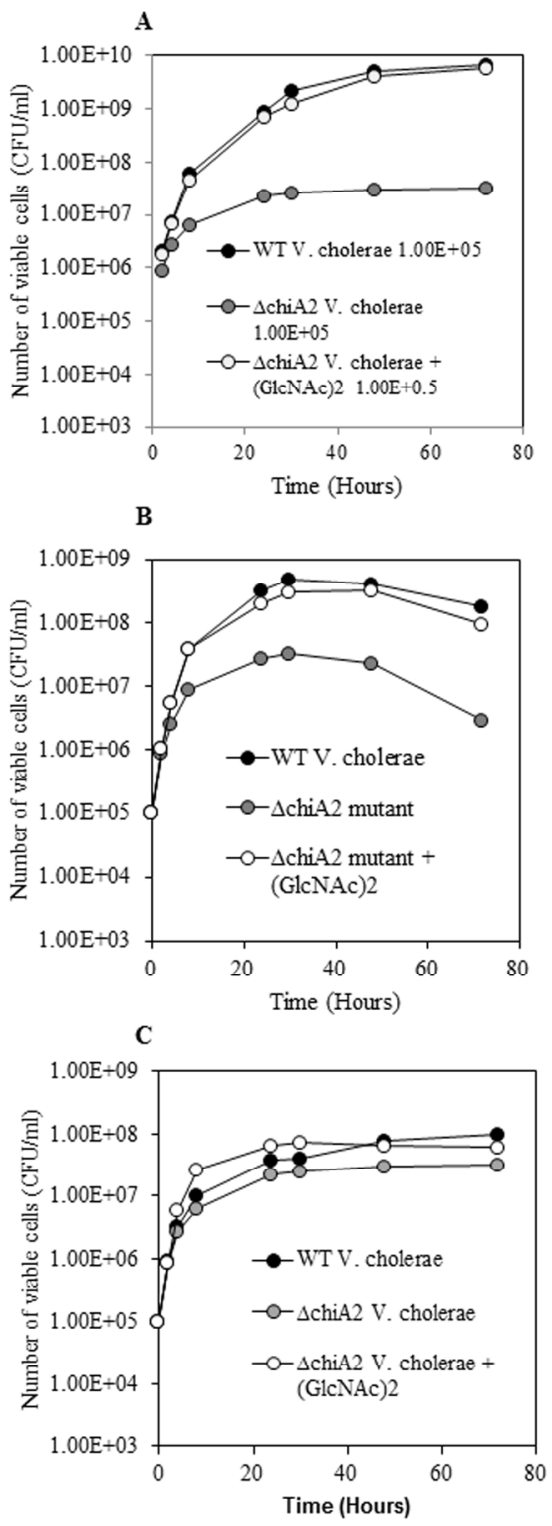

Figure 3,

Mondal et al., 2015

Figure 3. Graphical representation of the effect of environmental conditions on V. cholerae C6709 growth. Effect of environmental conditions on V. cholerae C6709 growth was studied in sea water media supplemented with $2 \%$ chitin separately under the mentioned three environmental conditions (Table 1 ). The growth curves were generated by plotting the number of viable cells (CFU/ml) in Y-axis again the time interval plotted in X-axis. The wild type V. cholerae $\mathrm{C} 6709$ was cultured in sea water media supplemented with $2 \%$ chitin separately under the three mentioned environmental conditions (Table 1 ). The number of viable cells after the time intervals $(0 h, 2 h, 4 h, 8 h, 10 h, 12 h, 24 h, 30 h, 48 h$ and $72 h)$ was enumerated by plate count method using TCBS plates. The $\triangle$ chiA2 V. cholerae was cultured in sea water media supplemented with $2 \%$ chitin or $6 \mathrm{mM}$ (GlcNAc)2 and was grown separately under the three mentioned environmental conditions (Table 1). The number of viable cells after the time intervals $(0 \mathrm{~h}, 2 \mathrm{~h}$, $4 \mathrm{~h}, 8 \mathrm{~h}, 10 \mathrm{~h}, 12 \mathrm{~h}, 30 \mathrm{~h}, 48 \mathrm{~h}$ and $72 \mathrm{~h}$ ) was enumerated by plate count method using TCBS plates. Figure presents the growth curve of V. cholerae C6709 wild type and the $\triangle \mathrm{chiA2}$ strains under A. condition I, B. condition II, C. condition III. 
$142 \times 254 \mathrm{~mm}(300 \times 300 \mathrm{DPI})$ 
를

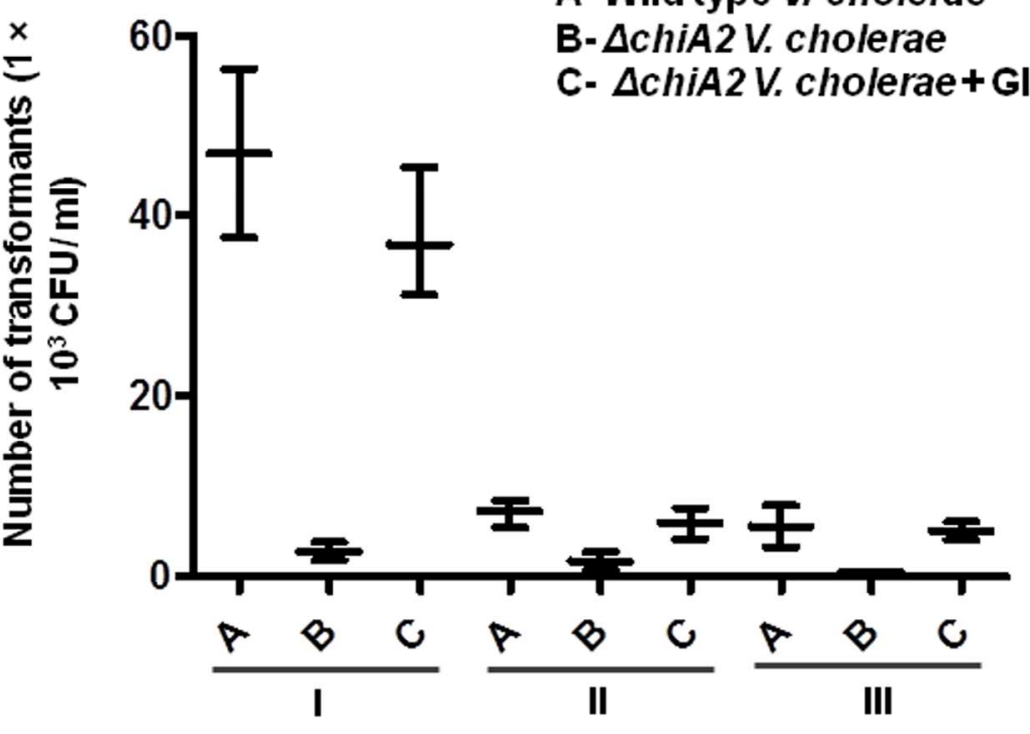

\section{A-Wild type $V$. cholerae \\ B- $\triangle$ chiA2 V. cholerae}

Figure 4,

\section{I- environmental condition I}

Mondal et al, 2015

\section{II- environmental condition II}

\section{III- environmental condition III}

Figure 4. Graphical representation of the effect of environmental conditions on V. cholerae Horizontal Gene transfer (HGT). Effect of environmental factors on V. cholerae HGT was studied in sea-water media supplemented with chitin or (GICNAC)2. $1 \times 109$ CFU of overnight cultures of V. cholerae and $\Delta$ chiA2 V. cholerae were inoculated with sea-water media and the transformation reaction was performed under three different environmental conditions (Table 1) with $3 \mu \mathrm{g} / \mathrm{ml}$ transforming ampicillin resistant GFP plasmid. The graph shows that condition I is the most favorable for $\mathrm{V}$. cholerae HGT compared to the other two conditions. However, the $\triangle$ chiA2 V. cholerae did not show HGT under any of the conditions except in presence of (GICNAc)2. The experiment was performed thrice and the data presented as mean \pm SEM. $170 \times 154 \mathrm{~mm}(300 \times 300 \mathrm{DPI})$ 
